\section{Programs for the analysis of dependencies in parent-child interaction sequences}

\author{
TERRENCE TAERUM, CON FERRIS, \\ HUGH LYTTON, and WALTER ZWIRNER \\ University of Calgary, Calgary, Alberta, Canada T2N $1 N 4$
}

The function of the programs described here is to analyze sequences of coded statements describing the interactive behavior of parents and their children. Their specific purpose is to identify antecedent actions which significantly influence the probabilities of certain behaviors occurring. Hayes-Roth and Longabaugh (1972) and Nirenberg, Haber, and Moise (1973) provide some guidelines for structuring the programs, but for our purposes the program should: (1) be able to use the Parent-Child Interaction Code, PACIC, which is a modification of APPROACH (Caldwell, 1969); (2) permit superordinate categories of psychological interest to be developed, e.g., compliance (Lytton \& Zwirner, 1975); (3) be selective in events chosen, since the number of possible combinations of subject-verb-object-modifier can be excessive even using the $r$ digits proposed by Nirenberg et al. (1973); (4) be selective in choosing events of psychological interest and events which could test hypotheses; and (5) be able to make use of time markers in order to limit the search for antecedents and successors within given time constraints.

The PACIC (Lytton, 1973) consists of behavioral statements of the form $\mathrm{CO2M} 5 \mathrm{C}$, meaning Child (C) goes to (02) Mother (M) quickly (5) in compliance with her request $(C)$. A short sequence might read:

$$
\mathrm{M} 71 \mathrm{C}-\mathrm{C} 02 \mathrm{M} 5 \mathrm{C}-1 \mathrm{C} 53 \mathrm{M}-\mathrm{M} 54 \mathrm{C}-\mathrm{M} 12 \mathrm{C}-1 \mathrm{C} 12 \mathrm{M} 41-\text {. }
$$

Each 6-place statement denotes an action by mother or child, the " 1 " in column 9 of some fields denotes a 10 -sec interval, which is used as a delimiter for various operations of the programs.

Two main programs were developed to analyze the statements: SEQ2, which carries out a two-stage analysis, and SEQ3, which carries out a three-stage analysis. All other programs use the output of these two programs to calculate conditional and unconditional probabilities.

\section{SEQ2}

SEQ2 is the most flexible program. It allows the user to specify grouped or single antecedents and successors, the subject of the antecedent and the successor, and the number of time intervals the program can look ahead for a successor. The program follows a sequence of actions

This research was supported by Canada Council Grant S 71-1770. Con Ferris is now at Red Deer College. searching for a specified antecedent and specified successors. The program then computes the unconditional probability (base rate), $\mathrm{p}\left(\mathrm{r}_{\mathrm{j}}\right)$, and the conditional probability, $p\left(r_{j} \mid a_{h}\right)$, and then evaluates the difference for significance (Bobbitt, Gourevitch, Miller, \& Jensen, 1969; Goodman, 1965).

The subject of the antecedent and the successor is always specified, whether it is for mother (M), father (F), or child (C). The vector of actions $R$ (successors) is always specified, but the vector of actions A (antecedents) may be grouped or left unspecified. Up to 20 single successors may be specified, and the computer can search for up to 52 possible antecedents. Grouped actions refer to individual verbs which the program has classified into superordinate groups.

The search for $r_{j}$ after an $a_{h}$ is limited by a specified time limit, which in all cases is two time intervals following the interval in which the $a_{h}$ occurred. The search forward is terminated by a " $Z$ statement," which indicates a change of setting.

As an example, we specified as vector $R$ the following verbs indicating attachment to mother: $\mathrm{C} 02 \mathrm{M}, \mathrm{CO} M \mathrm{M}$, and $\mathrm{C} 08 \mathrm{M}$. The program then searched for any mother action directed toward the child (verbs from MOOC to M77C) which were treated as antecedents to the child's actions. These verbs were also classified into the following classes to form grouped antecedents:

$a_{1}$ : Control-Command, prohibition,

$a_{2}$ : Control-Suggestion, request permission,

$a_{3}$ : Control-Command with justification (reasoning),

$a_{4}$ : Physical control,

$a_{5}$ : Negative action,

$\mathbf{a}_{6}$ : Positive action,

$a_{7}$ : Neutral action,

$a_{8}$ : No recorded antecedent action.

Search Algorithm for Two-Stage Analysis. Assume there exists a set of possible antecedents:

$$
A=\left(\begin{array}{llllllll}
a_{1} & a_{2} & a_{3} & a_{4} & a_{5} & a_{6} & a_{7} & a_{8}
\end{array}\right)
$$

and a set of possible successors:

$$
R=\left(\begin{array}{lllll}
r_{1} & r_{2} & r_{3} & r_{4} & r_{8}
\end{array}\right)
$$

where $a_{8}=$ no antecedent specified and $r_{8}=$ no response previously specified.

Given the following as an example of a fictitious schematized observation sequence (")" indicates the end of a 10-sec time interval):

$$
a_{1} a_{2} r_{1}\left|a_{2} a_{7}\right| r_{8} r_{8}\left|r_{8} r_{2}\right| r_{2}\left|a_{2} a_{7}\right| r_{8}\left|r_{1}\right| \text {. }
$$

The following dyads result: $a_{1} r_{8}, a_{2} r_{1}, a_{2} r_{8}, a_{7} r_{8}$, $a_{7} r_{8}, a_{7} r_{8}, a_{7} r_{2}, a_{8} r_{2}, a_{2} r_{8}, a_{7} r_{8}, a_{7} r_{1}$. 
The first sequence to analyze is:

$$
a_{1} a_{2} r_{1}\left|a_{2} a_{7}\right| r_{8} r_{8} \mid \text {. }
$$

The first $a_{h}$ is $a_{1}$. Because another member of A immediately follows, we assume no response, and $a_{1} r_{8}$ results. $a_{h}$ then becomes the next antecedent, which is $a_{2}$ followed by the successor $r_{1}$ within two time intervals, resulting in the dyad $a_{2} r_{1}$.

The search then continues after $r_{1}$. Since we pass a time marker, the next sequence to be analyzed becomes:

$$
a_{2} a_{7}\left|r_{8} r_{8}\right| r_{8} r_{2} \mid \text {. }
$$

Using the previously specified procedure results in the dyads $a_{2} r_{8}, a_{7} r_{8}, a_{7} r_{8}, a_{7} r_{2}$. Note that $a_{7}$ acts as an antecedent for all the responses until a different antecedent is recorded. This is because the question is: "What is the antecedent to different actions?" Hence, the antecedent to each of these successors is the same action, $a_{7}$. The search continues from the end of these successors.

The program then prints out frequencies of actions, unconditional probabilities of successors and antecedents, conditional probabilities of successors (given that specified antecedents have occurred), the probability that the conditional and unconditional probabilities are the same, and the joint probabilities of the successors and antecedents.

\section{SEQ3}

SEQ3 was written specifically for analyzing compliance sequences. It follows a sequence of actions over three stages, in which the action for the first stage is selected from set $A$, the action for the second stage from set $B$, and the action for the third stage from set $R$. The program classifies the raw coded statements of the input into predetermined categories as follows:

Vector A (prior antecedents): Categories $a_{4}$ to $a_{8}$ as defined in SEQ2.

Vector B (forms of verbal control):

$b_{1}$ : Mother (father) Command, prohibition.

$b_{2}$ : Mother (father) Suggestion, request permission.

$\mathrm{b}_{3}$ : Mother (father) Command with justification (reasoning).

$b_{4}$ : Mother (father) No form of verbal control.

Vector R (child successors):

$r_{1}$ : Child comply.

$\mathrm{I}_{2}$ : Child noncomply.

$r_{3}$ : Child neither comply nor noncomply.

The search for $a b_{i}$ action following an $a_{h}$ action is limited by the user-specified time interval, and similarly for an $r_{j}$ action following a $b_{i}$ action. The program computes conditional and unconditional probabilities in a similar way to the two-stage program SEQ2.

\section{SEQ4}

SEQ4 was written to analyze the relation between the prior antecedents developed in SEQ3 and the child successors of compliance, noncompliance, and neither. Given the set of $\mathbf{A}$ actions and $\mathbf{R}$ successors (previously defined), the program computes the conditional and unconditional probabilities by summing across the complexes set up on SEQ3.

\section{SEQSUM}

The function of the SEQSUM programs is to selectively sum over different sets of subjects to determine the conditional and unconditional probabilities of different behaviors within classification groups. For example, it is possible to derive estimates of the probability of actions for monozygotic and dyzygotic twins in order to compare the two groups. These programs allow the experimenter to carry out comparisons between subpopulations which appear to be of interest without having to run the more costly SEQ2 and SEQ3 again.

\section{Probability of Differences Between Probabilities}

The program calculates the probability of no difference between the conditional and the unconditional probabilities using a formula derived from Goodman (1965). A similar formulation and a set of procedures were used by Bobbit et al. (1969), and are contained in a description of the computer programs for that article. ${ }^{1}$

\section{Hardware and Software}

The programs were run on the CDC 6400 using up to $102 \mathrm{~K}$ of core for SEQ2 and 65K of core for SEQ3. SEQ2 requires 2,200 sec and SEQ3 2,000 sec to analyze approximately 50,000 cards for 136 children. The data for SEQ2 and SEQ3 are PACIC statements read off a tape. All other programs use the output from SEQ2 and SEQ3.

\section{Availability}

Details of the programs and their listings are obtainable at cost from T. Taerum, Department of Educational Psychology, University of Calgary, Calgary, Alberta, Canada.

\section{REFERENCES}

Bobitt, R. A., Gourevitch, V. P., Miller, L. E., \& Jenses, G. D. Dynamics of social interactive behavior: A computerized procedure for analyzing trends, patterns, and sequences. Psychological Bulletin, 1969, 71, 110-121.

Caldwell, B. M. A new "APPROACH" to behavioral ecology. In J. P. Hill (Ed.), Minnesota symposia on child psychology (Vol. 2). Minneapolis: University of Minnesota press, 1969.

Cochran, W. G. Some methods for strengthening the common $\chi^{2}$ tests. Biometrics, 1954, 10, 417-451.

GooDman, L. A. On simultaneous confidence intervals for multinomial proportions. Technometrics, 1965, 7, 247-254.

Hayes-Roth, F., \& Longabaugh, R. REACT: A tool for the 
analysis of complex transitional behavior matrices. Behavioral Science, 1972, 17, 384-394.

LYTTON, H. Three approaches to the study of parent-child interaction: Ethological, interview, and experimentation. Journal of Child Psychology and Psychiatry, 1973, 14, 1-17.

LYTTON, H., \& ZWIRNER, W. Compliance and its controlling stimuli observed in a natural setting. Developmental Psychology, 1975, 11, 769-779.

Nirenberg, L. M., Haber, J., \& Moise, S. L. A high-speed algorithm for computing conditional probabilities of substrings of sequentially observed data. Behavior Research Methods \& Instrumentation, 1973, 5, 291-294.

\section{NOTE}

1. Details of the programs and their listings by R. A. Bobbit, L. E. Miller, and M. J. Healy in Computer programs for interaction development research project were obtained from the Computer Library, Computer Center, University of Washington, Seattle, Washington, 98105. 